\title{
Iron acquisition mechanisms of the Burkholderia cepacia complex
}

\author{
Mark S. Thomas
}

Published online: 12 June 2007

(C) Springer Science+Business Media B.V. 2007

\section{Erratum to: Biometals \\ DOI 10.1007/s10534-006-9065-4}

We regretfully have to inform you that during the publication process of above-mentioned article, certain corrections to figure 3 have not been properly incorporated.
Please find below the corrected figure, with figure caption, as it should have been displayed. We would like to express our apologies for the inconvenience caused.
The online version of the original article can be found under doi:10.1007/s10534-006-9065-4.

M. S. Thomas $(\bowtie)$

Unit of Infection and Immunity, School of Medicine and Biomedical Sciences, University of Sheffield, Beech Hill Road, Sheffield S10 2RX, UK

e-mail: m.s.thomas@shef.ac.uk 

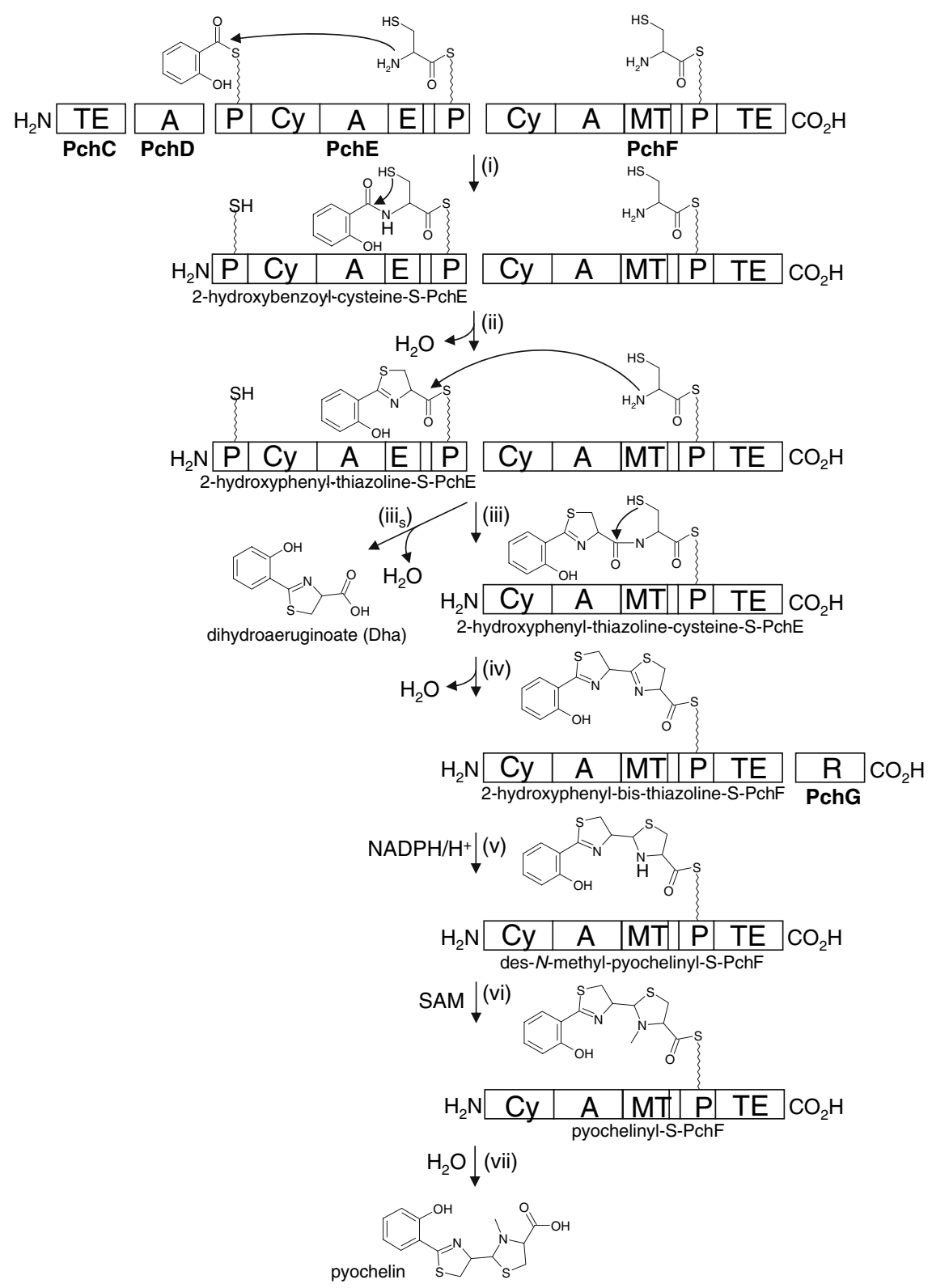

Fig. 3 Biosynthesis of pyochelin. In the first step, salicylic acid and two molecules of cysteine are activated by adenylation, then transferred to the phosphopantetheinyl arms (zigzag motifs) attached to the $\mathrm{P}$ domains of PchE and PchF. Correct loading of the $\mathrm{P}$ domains is ensured by the PchC thioesterase. Synthesis of the first intermediate is catalysed by the PchE Cy domain, and involves nucleophilic attack by the amino group of the PchEtethered cysteine on the thioester carbon atom of the salicylate group [step (i)], followed by cyclisation of the cysteine moiety and epimerisation to give rise to a mixture of D- and L-forms of the thiazoline ring [step (ii)]. This intermediate can give rise to Dha if it is hydrolysed off PchE [step (iii ${ }_{\text {s }}$ ] . Synthesis of the core pyochelin structure requires the PchF Cy domain, and occurs through nucleophilic attack by the amino group of the
PchF-linked cysteine on the thioester carbon atom of the Dha moiety [step (iii)], followed by cyclisation of the cysteine moiety [step (iv)]. The nascent thiazoline ring is reduced to a thiazolidine through the activity of PchG [step (v)] and methylated on the ring nitrogen [step (vi)]. The completed pyochelin molecule is hydrolytically removed from PchF by the C-terminal thioesterase domain [step (vii)]. A adenylation (activation) domain, $C y$ condensation and cyclisation domain), $P$ phosphopantetheinylation domain [also known as thiolation $(T)$, or aryl/peptidyl carrier protein $(A r C P / P C P)$ domain], $E$ epimerase domain, $M T$ methyl transferase domain, $R$ reductase, $T E$ thioesterase domain, $S A M$ S-adenosyl-methionine. Note that the epimerase and methyl transferase domains are located within the corresponding adenylation domains 